\title{
FE, RAZÓN Y HERMENÉUTICA EN EL PENSAMIENTO DE LOS JUDIOOS HISPANOS
}

\author{
Ángel Sáenz-Badillos \\ Universidad Complutense de Madrid
}

\begin{abstract}
RESUMEN
La historia de la filosofía judía dintingue dos grandes tendencias dentro de los judíos que vivieron en la península ibérica: la filosofía y la cábala. Sin embargo, creo que se deben tener en cuenta otros factores, como la cultura y la religión musulmana, la convivencia con la cultura cristiana y el contacto con los judíos europeos que vivieron una problemática parecida a la de los judíos españoles.
\end{abstract}

\begin{abstract}
Faith, reason and hermeneutic in the thought of The Spanish Jews. The history of the Jewish Philosophy differenciates two big tendencies among the Spanish Jews in the Middle Ages: the philosophy and the cabbala. However I think that we must take into consioderation other aspects such as the mussulman culture and religion, the living together with the Christian culture and the contact with the European Jews whose problem was similar to the difficulties of the Spanish Jews.
\end{abstract}

Si buscamos un hilo conductor en el pensamiento judío medieval, tal vez no encontremos otro más representativo que el de la relación entre la fe y la razón. No porque sea original ni privativo de los judíos, ni tampoco porque éstos adopten una actitud más o menos uniforme ante ese problema, sino porque de una u otra forma, todo pensador judío medieval tiene que comenzar a elaborar su sistema filosófico tomando postura ante esa cuestión fundamental. Por otra parte, y tratándose de un pensamiento que siempre tiene como punto de referencia un texto escrito, la Torah, el problema de la interpretación de ese texto en sus diversos niveles constituye uno de los temas básicos para cualquier pensador. ${ }^{1}$

1 Véanse a este respecto las palabras de C. Sirat: «Ainsi, on peut dire que l'histoire de la philosophie juive au Moyen Age est l'histoire de l'effort des Juifs pour concilier la philosophie (ou un système de pensée rationaliste) et le texte révélé. Selon les diverses philosophies, cet effort sera plus ou moins réussi; les différents éléments, philosophiques ou religieux, auront plus ou moins d'importance, mais l'harmonisation de ces deux systèmes de pensée en une vérité unique est le thème de presque toute la philosophie juive médiévale... Sans doute, pourrait-on d'ailleurs définir les philosophies médiévales, aussi bien arabes que chrétiennes, comme une réflexion sur les rapports entre la foi et la raison». La Philosophie Juive au Moyen Age, Paris, 1983,18. 
Dentro del pensamiento judío en la península ibérica se han distinguido y aun contrapuesto dos grandes manifestaciones, corrientes o tendencias: la filosófica y la cabalística. Y se ha elaborado un esquema un tanto simplista, según el cual, la filosofía sería predominante en todo el periodo andalusí que, culturalmente hablando, se extiende desde mediados del s. X (califato de `Abd al-Rahmān III) hasta mediados del s. XII (llegada de los almohades), mientras que la Cábala habría ocupado su lugar en la España cristiana a partir de comienzos del s. XIII y hasta la expulsión, continuando su actividad después de esa fecha en centros tan importantes como el de Safed. Aunque en esa perspectiva simplificada hay algunos puntos de verdad, se hace necesario precisar mucho más los matices para dejar las cosas establecidas en su justo término.

La cultura judía medieval en España sólo puede entenderse adecuadamente a partir de cuatro factores que la determinan: en primer lugar, el propio legado tradicional, el mundo de la Biblia y la literatura rabínica (Mišnah, Talmud, normas jurídicas judías) en el que todo judío medieval se encuentra profundamente enraizado; en segundo lugar, la cultura y el pensamiento árabe-musulmán, en el que se hallan inmersos los judíos peninsulares hasta que el avance de la Reconquista y las presiones de los grupos intransigentes norteafricanos producen la desbandada de las comunidades andalusíes; un tercer factor, quizá menos importante pero no despreciable, es el impacto de la convivencia con la cultura cristiana española y europea, al menos a partir del s. XII, cuando comienzan a cobrar fuerza las nuevas juderías de los territorios reconquistados; finalmente, el contacto con los judíos europeos, franceses, italianos o alemanes, que han vivido durante siglos una problemática muy distinta y se acercan a los judíos hispánicos comunicándoles sus experiencias y perspectivas. El pensamiento judío medieval en Sĕfarad depende en mayor o menor medida de varios de esos factores, y sin pretensiones de originalidad va elaborando su propia síntesis con avances $y$ retrocesos, con preferencias por una u otra línea ideológica, y casi nunca de manera unilineal o monolítica.

Hablamos de pensamiento judío, y expresamente hemos escogido esa formulación, porque hablar de una «filosofía judía» medieval podría parecer extraño a quienes por filosofía entienden ante todo un sistema cerrado y coherente. Para los que buscan esto último, habrá que decir que filósofos sistemáticos apenas se dan entre los judíos españoles, si exceptuamos algunas individualidades com Šĕlomoh ibn Gabirol. Y aun en esos casos, seguràmente quedarán decepcionados los que busquen algo realmente original, una síntesis que no dependa de las corrientes ideológicas de la época, y que aun estando impregnadas de cultura árabe, eran de origen claramente helenístico. Pero eso no significa que los judíos de Šfarad no se plantearan en muchos casos la búsqueda de la sabiduría, la ciencia y la razón, que están en la base del término mismo de «filosofía», como la tarea más importante de su vida. Ni que el problema de conciliar esa búsqueda con el carácter revelado de la Torah no fuera uno de los principales motivos de discordia entre los miembros de las comunidades judías hispanas a lo largo de la Edad Media. 
La controversia entre los defensores de la fe y de la razón se da en todo el Medievo, tanto entre los cristianos ${ }^{2}$ como entre los musulmanes ${ }^{3}$ y los judíos. ${ }^{4}$ Partiendo de presupustos bastante similares, los intentos de solución son asimismo semejantes entre los pensadores de las tres religiones, que tratan de interpretar racionalmente las que se consideran verdades reveladas (como al-Färäbî ), de buscar el acuerdo entre los principios de la verdad revelada y los resultados de la investigación racional (como harán, cada uno a su manera, Avicena, Averroes, Maimónides o Tomás de Aquino, así como buen número de escolásticos y de pensadores musulmanes y judíos), o bien de dar la total primacía a la revelación en detrimento de la razón (como al-Gazālì o Yěhudah ha-Levi).

Lo cierto es que los judíos se vieron obligados desde muy pronto a plantear esta cuestión, al menos desde el momento en que el encuentro con el helenismo, varios siglos antes de Cristo, exigió de ellos una clarificación sobre la pregunta de si la verdad absoluta se encontraba en la Torah, que consideraban recibida del mismo Dios, o en los libros de los filósofos griegos. En la literatura rabínica se encuentran con relativa frecuencia alusiones a lo que se llamaría la «ciencia de los griegos»-incluyendo la filosofía y las demás ciencias- como contrapuestas al propio legado de la tradición judía. ${ }^{5}$ A pesar de ello, y a partir del intento de conciliación entre fe y razón que se encuentra en la obra de Sẻ adyah Ga'on, no pocos judíos que viven en el área cultural del Islam, y particularmente en al-Andalus, desarrollan más y más la idea de que no solamente no se da una contradicción entre ese saber helenístico y la tradición religiosa judía, sino que la razón podía ser incluso un elemento necesario para tributar el verdadero culto a Dios y para acercarse a Él. En la escala de valor de la humanidad, los pensadores judíos sitúan a los filósofos en un lugar muy destacado, aunque al mantener intacta una perspectiva esencialmente religiosa, subrayarán siempre que es superior la categoría del profeta, el hombre que se acerca más a Dios y que llega a tratar directamente con Él.

Otra constante del pensamiento judío es la cuestión hermenéutica, consecuencia de su dependencia clara de un texto, el de la Torah, a la que se atribuye origen divino. Si los musulmanes sî̀ítas, en una situación similar, desarrollan con claridad la teoría dé que el texto sagrado tiene un sentido literal, el zāhir, y un sentido más profundo, el bățin, el pensamiento judío

\footnotetext{
2 Cf. É. Gilson, Reason and Revetation in the Middle Ages, New York, 1938

3 Cf. A. J. Arberry. Revelation and Reason in Islam, Londres, 1957. Véase también J. Lomba, La Filosofía islánica en Zaragoza, 1987:

4 Cf. J. Sarachek, Faith and Reason. The Conflict over the Rationalism of Maimonides, Williamsport, 1935; D. J. Silver, Maimonidean Criticism and the Maimonidean Controversy, 1180-1240, Leiden, 1965; I. E. Barzilay, Betwieen Reason and Faith. La Haya-París, 1967; Ch. Touati, «La controverse de 1303-1306 autour des études philosophiques et scientifiques», REJ, 127, 1968, 21-37; F. Talmage, «David Kimhi and the Rationalist Tradition». HUCA 39, 1968, 177-218; etc.

5 Cf. A. Sáenz-Badillos, J. Targarona: «La 'ciencia de los griegos" en los judíos de la España medieval», Homenaje al Dr: L. Gil Fernández; en prensa. No pocas veces se sugiere por parte de los pensadores medievales judíos que los sabios griegos aprendieron cuanto sabían de Moisés y los grandes Profetas de Israel, y que los rabinos escribieron sobre ciencia y filosofía: aunque sus escritos se hayan perdido por culpa del exilio. La idea no es nueva: los judíos de Alejandría como Aristóbulo, citado por Josefo y Eusebio, la sostenían ya en el s. Il a.C. Así se explicaba apologéticamente la ausencia de obras científicas en hebreo. La afirmación de que los israelitas enseñaron la ciencia a todos los pueblos se recoge, v.gr. en el Kuzari de Yěhudah ha-Levi, II, 66.
} 
medieval puede sumar a estas fuentes su propia tradición antigua, en la que el alegorismo fue muy pronto conocido. Junto al sentido literal o pěsat, y la exposición del texto elaborada por los procedimientos rabínicos o dĕraš, los filósofos medievales prefieren con frecuencia la alegoría, remez, mientras los cabalistas buscan el sod o sentido oculto y misterioso del texto. La situación no es muy distinta de la que se da entre los exegetas cristianos, que también conocen desde muy antiguo las distintas dimensiones del texto y son tradicionalmente tachados de «alegoristas» en los textos de controversia. ${ }^{6}$

Como ya indicábamos, se suele identificar la gran época de la filosofía judía en España con el periodo andalusí, lo que no es del todo inexacto. Sin embargo, no conviene olvidar que el acercamiento del judaísmo a la problemática básica de la filosofía tiene lugar en Oriente, y de modo particular con la figura de Sě̀adyah Ga'on, que en éste como en otros muchos terrenos fue un auténtico pionero de los avances medievales. Y, preludiando lo que pocos años más tarde sucederá en al-Andalus, S `` adyah se acerca a esta problemática planteada por los filósofos árabes, sin renunciar a sus profundas raíces religiosas dentro del judaísmo. En medio de un ambiente de polémica, Sě adyah es capaz de elaborar un sistema teológico racional que sirve de defensa a la Torah, responde a los ataques sectarios contra el judaísmo rabínico y demuestra su superioridad absoluta. ${ }^{7}$ La tradición auténtica, la de la Torah, es una de las vías de conocimiento del hombre, basada a la vez en el conocimiento de los sentidos y en el de la razón. Torah y ciencia son dos brotes de una misma rama, que no pueden contradecirse en nada; si se da un aparente enfrentamiento, es que no hemos razonado bien o que hemos interpretado mal el texto revelado. ${ }^{8}$

Sin duda, el Ga'on deja abierto un camino por el que podrán avanzar los judíos hispanos sin sentir desde el primer momento el rechazo y la condena de los partidarios exclusivamente de la propia tradición. La autoridad de quien fuera líder indiscutible de su generación servirá de base firme para los primeros judíos que en al-Andalus se atreven a aventurarse por esta vía. Pero tampoco era eso suficiente para que dejara de cuestionarse entre los más fervientes tradicionalistas hasta qué punto era legítimo para un judío salirse de sus propias categorías religiosas, largo tiempo establecidas y enseñadas, y buscar en el saber de otros pueblos algo útil y provechoso para su propia vida religiosa. El que la filosofía judía medieval tenga su mayor desarrollo durante la época andalusí no significa que todos los judíos la aceptaran en ese tiempo sin dificultad, mientras que en la España cristiana la rechazaran todos unánimemente. Hubo anti-racionalistas en el periodo andalusí, del mismo modo que se dan críticos de la tradición en la España cristiana. Pero desde el momento en que se creó en al-Andalus una cultura judía más o menos autóctona, bajo el impulso de Hasday ibn Šaprụ, la mayor parte de los intelectuales judíos no tendrían problema en cultivar la mejor tradición científica y filosófica de los griegos y los árabes, sin ver en ello peligro alguno para su propia fe y tradición judías.

6 Cf. Ios estudios clásicos de B. Smalley, The Study of the Bible in the Middle Ages, Oxford, 1952, y H. de Lubac, Exégèse Médiévale. Les quatre sens de l'Écriture, Lyon, 1959.

7 Cf. C. Sirat. op. cit., p. 35.

8 Op. cit., pp. $37 \mathrm{~s}$. 


\section{NEOPLATONISMO Y COMIENZOS DEL ANTI-RACIONALISMO}

Durante el s. XI y la primera mitad del s. XII, la corriente filosófica más extendida entre los judíos andalusíes es la neoplatónica. Dentro de ella, el malagueño Šĕlomoh ibn Gabirol (ca. 1020- ca. 1057), afincado en su juventud en Zaragoza, es seguramente el pensador judío medieval que mejor responde al calificativo tradicional de «filósofo», en cuanto autor de un sistema ontológico y cognoscitivo coherente. Lo desarrolla en su «Yanbui al-hayya», en árabe, que ha llegado hasta nosotros extractado en hebreo y traducido al latín como Fons Vitae por Juan Hispano y Domingo Gundisalvo (s. XII). ${ }^{9}$ Se trata de una obra inspirada en las doctrinas neoplatónicas entonces en boga, sin ninguna característica que permita definirla como elaborada por un pensador judío, ya que el autor plantea las cuestiones más elevadas de la naturaleza del universo y su relación con Dios sin ninguna connotación exclusivista. Ibn Gabirol cita a Platón, pero tiene además un fuerte influjo de Aristóteles entendido a través de los neoplatónicos, de Plotino y el Pseudo-Empédocles en particular; y se basa también en numerosos pensadores musulmanes y aun judíos, como Sĕ adyah o Yiṣhạq Iśraeli, pero sin recurrir en ningún caso a su propia tradición bíblica o rabínica. Ni siquiera se plantea como pregunta básica el problema de la relación entre la razón y la fe, como lo harán otros muchos de sus correligionarios en los siglos siguientes. Para él es simplemente evidente que no existe problema alguno entre ellas, y no se pone siquiera en cuestión que el Dios de la Biblia y el de los filósofos son uno solo.

Ibn Gabirol parte de las ideas neoplatónicas de que la ciencia es el fin supremo de la vida humana y su razón de ser, y de que el conocimiento que el hombre tiene de su alma le permite acceder al conocimiento del mundo y de Dios, puesto que él mismo es un microcosmos; el alma humana procede del mundo superior, y está llamada a volver a ese mundo mediante el conocimiento y la acción. El sistema ontológico va de arriba abajo: de las sustancias superiores o sustancias simples emanan o fluyen las inferiores de manera similar a la de la luz cuando se propaga por el espacio, debilitándose cada vez más. La esencia primera, Dios, actúa a través de su Voluntad, que es la que crea y da el ser a la materia y la forma universales, de las que proceden o son emanados todos los demás seres, compuestos de materia y forma. Así intenta conjugar la creación bíblica con la emanación neoplatónica. El camino del hombre consiste en la ascensión por la vía del conocimiento, de la reflexión sobre sí mismo y sobre el mundo, hasta esa Voluntad creadora, y hasta la Esencia primera, Dios mismo, alcanzando «la liberación de la muerte y la unión a la fuente de la vida» $(\mathrm{V}, 43)$.

9 El texto hebreo fue editado por S. Munk, Paris 1859; trad. franc. J. Schlanger, 1970, con buenas notas explicativas; versión española de F. de Castro, reed. Barcelona 1987. Véase J. Schlanger, La Philosophie de Salomon Ibn Gabirol, Leiden, 1968. Puede verse asimismo mi breve estudio El alma lastimada: Ibn Gabirol, El Almendro, Córdoba. 1992. Mayor información sobre los filósofos hispanojudíos que se mencionan en este trabajo puede encontrarse igualmente en: A. Sáenz-Badillos, J. Targarona Borrás, Diccionario de Autores Judios (Sefarad. Siglos X-XV), El Almendro, Córdoba, 1988. 
Ibn Gabirol lee la Escritura con ojos de fitósofo y, aunque no sabemos con qué extensión, aplica el método alegórico, ${ }^{10}$ encontrando en ella la confirmación de un sistema filosófico elaborado de forma independiente. Sin embargo, parece más preocupado por descubrir la coincidencia fundamental entre Biblia y metafísica, que en señalar que ese sentido filosófico es precisamente el que que quiere transmitir el texto bíblico más allá de su misma literalidad.

Bahya ibn Paqudah (ca. 1040-ca. 1110) el filósofo moralista de Zaragoza, aun teniendo otros intereses primarios, se preocupa también de la problemática de la fe y la razón de modo indirecto, reconociendo en la ciencia el don supremo de Dios a los hombres. Fe y razón se complementan: en el tema de los preceptos, al que dedica su atención preferente, la revelación precisa y completa los mandamientos que el hombre conoce ya por su razón natural. " Según él, el estudio de la metafísica es necesario para poder captar la religión.

El acuerdo entre la fe y la razón juega también papel no pequeño en el Microscosmos del neoplatónico cordobés Yosef ibn Șaddiq (ca. 1070-1149). Glosando las palabras de Jb 19,26 «desde mi carne veré al Señor», Ibn Saddiq sostiene que gracias a la facultad de razonar que el Creador ha dado al hombre, y mediante el conocimiento de su cuerpo y su alma, el hombre alcanza el conocimiento de Dios. La revelación no viene sino a completar el esfuerzo del hombre por conocer con su entendimiento, como ocurrió en el caso de Abraham. Al entregar Dios la Torah a Moisés le dió el don de la profecía, pero actualmente nadie puede alcanzar la profecía, que es la verdadera filosofía, sino es mediante el estudio de las ciencias. ${ }^{12}$

Para Abraham bar Hiyya' (ca. 1065-ca. 1143), la ciencia y la virtud que permiten al alma ascender hasta su verdadera patria celestial no pueden enseñarlas los filósofos, que no han recibido el don de la Torah; no obstante, el acuerdo entre la ciencia y la Torah es total, ya que la ciencia, e incluso la metafísica, la aprendieron los sabios de otras naciones de los grandes maestros de Israel. ${ }^{13}$ En su obra se presenta por primera vez en hebreo el pensamiento de Ptolomeo, junto a concepciones cosmológicas neoplatónicas y aristotélicas. Su esfuerzo de síntesis de estos conocimientos con la Escritura es evidente:

"Los sabios antiguios llaman a uno de ellos en lengua griega hywly (i.e., hylè), y dicen que que es algo que no tiene imagen ni forma, y está destinado y dispuesto a recibir imagen y toda forma. $Y$ al segundo le llaman forma, y dicen que es algo que tiene poder y fuerza para revestir a la hywly de toda imagen y forma... Y te encuentras con que todos sus. grandes han tomado esas cuestiones que han sido expuestas hasta este lugar de las palabras de la Torah, y sacan agua del manantial de la sabiduría, si te interesas por ellos. Y te encontrarás cen que la hywly y la forma, que según sus palabras existían en poten-

10 Cf. mi trabajo «La exégesis de la creación y el paraíso en la obra de Selomoh ibn Gabirol». en Palabra y vida. Homenaje a José Alonso Díaz en su 70 cumpleaños, ed. A.Vargas-Machuca y G.Ruiz, Madrid 1984, 369-382.

It Cf. G. Vajda, La teologia ascética de Bahia ibn Paqudo, Madrid-Barcelona, 1950; C. Sirat, op. cit. $73 \mathrm{~s}$.

12. Cf. C. Sirat; op. cit. $104 \mathrm{~s}$.

13 Cf. C. Sirat, op. cit., 114 s.; L. D. Stitskin, Judaism as a Philosophy: The Philosophy of Abraham Bar Hiya (1065-1143). New York: 1960. 
cia antes de la creación del mundo, son el tohu wa-bohu que era la tierra según testimonia la Torah...»."

Está convencido de que los judíos no deben dudar en estudiar las obras de los antiguos, ya que la Torah lo permite y aun lo ordena, si bien en último término «nuestra autoridad es la Escritura, y tenemos que comprobar las opiniones de los filósofos mediante las enseñanzas de la Biblia. ${ }^{15}$

Sin embargo; a pesar de esas posturas positivas, compartidas por buen número de intelectuales andalusíes, pronto se oyen en suelo andalusí voces contrarias a las ideas procedentes de fuera del judaísmo, defendiendo los que se consideran valores propios de su pueblo.

Yěhudah ha-Levi (ca. 1070-1141), formado en tierras castellanas, adopta una postura claramente contraria a la razón y la filosofía, que se expresa tanto en su obra poética como en su escrito apologético-filosófico, el Kuzari. La tradición es para él más segura que la razón para alcanzar la verdad en las cuestiones más difíciles y delicadas:

«Kuzari: ...Pero ¿qué me dirás de los filósofos, que llegaron muy arriba con su investigación y estudio? ¿No estaban de acuerdo en la idea de la eternidad y preexistencia del mundo, y la eternidad no es cosa de decenas de miles de años ni de miles de millares, sino de algo que no tiene fin?

Hạber: No hay que culpar de ello a los filósofos, puesto que se trata de gentes que no recibieron la sabiduría ni la Ley como herencia; fíjate que son griegos (descendientes de Yawan), y Yawan es de los hijos de Jafet que habitaban en el Norte, mientras que la sabiduría que fue dada come propiedad al primer hombre, esto es, la que se consigure gracias a la «capacidad divina», pasó de Adán únicamente a la descendencia de Šem, el escogido de los hijos de Noé, y desde entonces no cesó ni nunca cesará entre esos hombres escogidos. En cuanto a los griegos, no les llegó la sabiduría sino después de que fueran vencidas las naciones que lucharon contra ellos, pues fue en ese momento cuando les pasó a ellos la sabiduría de los persas, que la habían recibido de los caldeos; sólo entonces surgieron los filósofos famosos de ese imperio; y más aún, desđe que pasó el poder a Roma no surgió entre los grieges ni un solo filósofo famoso más.

Kuzari: ¿Acaso esto nos obliga a no creer en la sabiduría de Aristóteles?

Haber: Así es, y eso se debe a que Aristóteles no poseía una tradición fiel de hombres que hubieran tratado con verdad el tema, y tuvo que fatigar su entendimiento y ejercitar su mente investigando el principio y el fin del mundo. Le resultaba difícil imaginarse que el mundo tiene principio, igual que le resultaba difícil representarse la eternidad del mundo, y únicamente con su estudio abstracto se inclinó del lado de los argumentos que favorecen la eternidad. Por eso no vio la necesidad de preguntar acerca de las generaciones que le precedieron, ni de cómo fureron los linajes de los hombres. Pero si el filósofo hubiera pertenecido a una nación en la que nociones verdaderas e irrefutables se transmiten por tradición, habría empleado sus razones y argumentos para

14 Sefer Hegion ha-Nefesch oder Sitten-Buch. ed. E. Freimann. Leipzig. 1860, f. Ib s.

15 Op. cit. 5 a. 
confirmar la creencia en que el mundo fue creado, con todas sus dificultades, del mismo modo que lo hizo tratando de probar la eternidad del mundo, mucho más difícil de aceptar. Kuzari: ¿Qué prueba concluyente puede haber donde hay un argumento lógico?

Hạber: ¿Se ha encontrado alguna vez un argumento en esta cuestión? ¡No quiera Dios que haya nada en la Torah que contradiga el testimonio de algo que se ve con los ojos o que se prueba con un argumento racional! No obstante, se mencionan en la Torah milagros en los que hay cambio del orden originario, bien sea creando nuevas criaturas, o cambiando una en otra, pero todas esas cosas no vienen sino a probar que el Creador del mundo puede hacer a su voluntad cuanto desea siempre que quiere. La cuestión de la eternidad del mundo o de su creación es difícil de resolver, y los argumentos en favor de ambas están equilibrados. Lo que inclina la balanza a favor de la creación es la tradición de Adán, Noé y Moisés, el testimonio de la profecía que es más fiable que el del argumento lógico. Y con todo, si alguien que cree en la Torah se convence racionalmente de la noción de la eternidad de la materia unida a la idea de que a este mundo nuestro le precedieron otros muchos mundos, no habría nada en contra de su fe en que este mundo nuestro fue creado hace un tiempo determinado, y que sus primeros hombres fueron Adán y Noé». ${ }^{16}$

Según él, los judíos no necesitan la especulación filosófica, porque han recibido de Dios un conocimiento más elevado: por encima de la vía de los filósofos está la de los profetas, a los que el mismo Dios ha concedido una especie de «capacidad divina» (Amr llähì) que es muy superior a la razón humana. Por otra parte, se da una oposición fundamental entre la religión revelada por excelencia, el judaísmo, y la filosofía, ya que ésta llega únicamente a un concepto abstracto e impersonal de Dios, mientras que el Dios que se revela tiene su voluntad propia y personal. ${ }^{17}$

YHL no discute directamente cuestiones de hermenéutica, pero al condenar los intentos de acercamiento entre filosofía y religión rechaza todos los instrumentos que podrían ayudar a esa conciliación, y entre ellos, la exégesis alegórica que puede tratar de cambiar el sentido primario de un texto. ${ }^{18}$

Probablemente, la obra de Yěhudah ha-Levi es la primera expresión sistemática de un tipo de anti-racionalismo perfectamente articulado y justificado, que se convertirá en los siglos siguientes en una de las corrientes dominantes del pensamiento judío en Sĕfarad. ${ }^{19} \mathrm{Su}$ firme posición anti-filosófica tiene su paralelo en la obra del pensador musulmán Al-Gazāli ${ }_{s}{ }^{20}$

16 Kuzari I, 62-7.

17 Cf. M. R. Hayoun, L'exégèse philosophique dans le judaïsme médiéval, Tübingen, 1992, 124.

18 Cf. M. R. Hayoun, op.cit., 128.

19 Cf. B. Septimus, Hispano-Jewish Culture in Transition. The Career and Controversies of Ramah, Cambridge/Mass., p. 62.

20 Cf. D. Z. Baneth, «Jehuda hallewi und Gazăli», Wissenschaft des Judentums im deutschen Sprachbereich. Ein Querschnitt. Tübingen, 1972, 371-389; M.R. Hayoun, op.cit. 125. El ataque de al-Gazālì, contra la filosofía fue aún peor que el de YhL. En su Tahafot al-Falasifa declara a los filósofos culpables de herejía porque no creen en la resurrección de los cuerpos ni en la omnisciencia de Dios. Por lo demás, en no pocos detalles, Yěhudah haLevi sigue bastante de cerca al pensador musulmán. 
Abraham ibn 'Ezra', tudelano también (1089-1164), y estrechamente relacionado por diversos motivos con Yěhudah ha-Levi, adopta una postura bien distinta: la revelación no puede según él sustituir al esfuerzo intelectual del hombre. ${ }^{21}$ «Las ciencias son muchas, y todas ellas son útiles, y son como peldaños de la escala para subir hasta la verdadera ciencia», dice en su Sefer Yěsod Mora' ${ }^{22}$ Al comienzo de esta obra distingue diversos tipos de sabios en Israel (expertos en masora, en gramática, en la Biblia, en el Talmud), y hace notar que sin un conocimiento de geometría, astronomía, psicología, fonética, etc., no podrá nadie conocer verdaderamente «las obras del Señor», ni interpretar adecuadamente los textos bíblicos..$^{23}$ Respecto a estos últimos, Ibn 'Ezra’ reconoce que el sentido literal siempre es auténtico, pero que en algunos lugares también lo es el sentido más profundo del texto. ${ }^{24}$

\section{EL ARISTOTELISMO}

A mediados del s. XII el neoplatonismo cede su puesto a la filosofía de cuño aristotélico. Ese hecho contribuye a que se manifieste con la mayor fuerza la actitud pro-racionalista de algunos de esos intelectuales, y al mismo tiempo, provoca la reacción contraria de quienes ven en esa postura un peligro grave para la tradición de sus mayores. Las tensiones llegan a su punto extremo en el siguiente siglo, y ya no desaparecen de la sociedad judía hasta la salida de España.

Uno de los primeros pensadores judíos sefardíes que desde esa nueva perspectiva plantea la cuestión del acuerdo entre filosofía y Torah es Abraham ibn Daud, que escribe en 1160/1 su obra filosófica en árabe traducida al hebreo con el nombre generalizado de Ha-'ěmunah haramah, «La fe excelsa». Según sus primeras palabras, «presenta el acuerdo entre la filosofía y la religión». Ibn Daud no tiene reparo en señalar que las palabras del Sal 139,12, «no es oscura la tiniebla para $\mathrm{Ti}$, pues ante $\mathrm{Ti}$ la noche brilla como el día», son una referencia a la jerarquía de las luces, en cuya cima se encuentra el conocimiento de Dios. ${ }^{25}$ En su opinión, el judío no puede tener una plena formación sin conocer además de las ciencias tradicionales todas las de la sabiduría griega. ${ }^{26} \mathrm{Se}$ da una coincidencia fundamental entre la fe revelada y la filosofía, que sólo puede entenderse plenamente desde las categorías aristotélicas. Sus demostraciones filosôficas van siempre corroboradas con citas bíblicas interpretadas en sentido literal o, en caso de necesidad, alegórico. Cuando en algún punto no parece haber acuerdo entre la razón y la fe, procura buscar una solución de compromiso:

\footnotetext{
21 Cf. C. Sirat, op. cit. 122 ss.

22 Ed. l. Levin, Abraham ibn Ezra Reader, 1985, 315.

23 Op. cit.. $315 \mathrm{ss}$.

24 Cf. M.-R. Hayoun, L'exégèse philosophique dans le judaïsme médiéval, Tübingen, 1992, 147 ss.

25 Cf. T. A. M. Fontaine, In Defence of Judaism: Abraham Ibn Daud. Sources and Structure of ha-Emunah ha-Ramah. Assen/Maastricht. 1990.

26 Cf. J. Targarona, «Pensadores judíos cordobeses de los ss. XI-XII: Yosef ibn addiq. Abraham ibn Daud y Mošeh ben Maimon», en Los Judios y Lucena, ed. J. Peláez, Córdoba, 1988, 49-64.
} 
«Y puesto que la Torah y la filosofía están en contradicción en ese punto, nos vemos como quien tiene dos señores, uno de ellos grande, y otro no pequeño, y no hay manera de complacer a uno de ellos si no es contra el parecer del segundo; si encontramos modo de establecer un acuerdo entre ellos, se nos reputará como un éxito». ${ }^{27}$

Al filósofo cordobés Mošeh ben Maimon, Maimónides (1138-1204), se debe el mayor esfuerzo por conciliar la filosofía y la razón por un lado y la fe judía por otro. Con un gran afán de integración, trata de hacer ver a sus correligionarios que la búsqueda de la verdad mediante el estudio de la lógica, la física y la metafísica podía armonizarse sin serios inconvenientes con la práctica del judaísmo. ${ }^{28}$ Pocos pensadores judíos del Medievo contribuyen de manera similar a eliminar las posibles contradicciones o problemas entre fe y razón. En la Introducción de la Guía de Perplejos escribe:

«La razón de ser del presente tratado... es el estudio científico de la Torah en su auténtico sentido, o más bien ilustrar al hombre religioso en cuya alma está anclada la verdad de nuestra Ley como objeto de creencia, que se muestra perfecto en su religiosidad y costumbres, ha estudiado las ciencias filosóficas y conoce sus secretos, y al cual la razón humana atrae y guía a sus dominios, pero se encuentra desorientado por la exterioridad de la Torah... reducido a un estado de perplejidad y confusión: 0 adherirse a lo que al respecto aprendió conforme a su saber y entender, y entonces se imaginaría haber traicionado a los fundamentos de su Ley, o bien atenerse a lo que captó sin dar entrada al raciocinio; en tal supuesto habría renunciado a la razón, alejándose de ella, convencido de un menoscabo y pérdida en su religión, y obstinado en semejantes fantasías, será presa de inquietud y congoja, con el corazón atenazado y violenta turbación».9

En esta obra fundamental trata de hacer la síntesis del saber tradicional judío, que durante siglos ha especulado sobre la creación y la manifestación de Dios, y las teorías filosóficas de la Antigüedad. Según sus conocidas palabras:

«En mis escritos talmúdicos... advertí que el Ma ‘ŭseh bĕ-re’sit («obra, o relato, de la Creación») se identifica con la Física, y el Ma ăšh merkabah («relato de la Carroza celeste»), con la Metafísica». ${ }^{30}$

Ambos temas se encuentran en el fondo de los «secretos y misterios de la Torah», que serán objeto muy especial de su atención. Eso significa que, en su opinión, los contenidos de la filosofía aristotélica y los de la fe mosaica coinciden en su esencia; pero, al mismo tiempo,

27 Sefer ha'ěmuna 
que frente a las especulaciones un tanto vacías de sentido de los rabinos centroeuropeos, el judío debe encontrar en el estudio de los filósofos, y, particularmente de Aristóteles, el camino para profundizar en su fe. ${ }^{31}$ Esa consideración racional de los «secretos de la Torah» será uno de los temas que resultarán más escandalosos para los defensores de la tradición judía de su época, provocando fuertes reacciones.

Preocupado por lo que puede servir de tropiezo a quienes se sienten judíos pero conocen también la filosofía y las ciencias, Maimónides enumera las causas de contradicciones u oposiciones que pueden aparecer en cualquier tipo de escritos, incluidos los bíblicos. Para el caso concreto de estos últimos sugiere que las proposiciones aparentemente contradictorias pueden tener sentido literal en un caso y alegórico en el otro, o se deben a la falta de concreción en uno de los dos pasajes, haciendo que parezca que hay una contraposición, cuando en realidad no es ése el caso. Para eliminar los obstáculos del camino y penetrar así en los «misterios de la Torah» recurre a la exegesis alegórica. Cuando en uno de esos textos tradicionales, y particularmente en los bíblicos, se encuentra algo que parece ir en contra de la razón, o es un enigma o una alegoría.

De acuerdo con un principio pedagógico, para no desconcertar a las masas incultas, las secciones más trascendentes de la Escritura están redactadas en forma de parábola, con un sentido externo, aparente, que puede llegar incluso a ocultar su verdadero significado, el sentido interno del texto. Es como una perla perdida entre los muebles de una casa a oscuras; solamente encendiendo la luz -comprendiendo el sentido de la parábola- es posible encontrar la perla y disfrutar de ella:

«el sentido interno de las palabras de la Torah es una perla, mientras que el sentido externo de las parábolas no vale nada». ${ }^{32}$

Es como una «manzana de oro cubierta por una filigrana de plata» $(\operatorname{Pr} 5,11)$ que deja entrever a través de sus calados el brillo escondido en su interior.

«Las parábolas de los Profetas... son algo similar. Su sentido externo contiene sabiduría útil en muchos aspectos... Su sentido interno contiene sabiduría útil para las creencias relativas a la auténtica verdad».33

Los ignorantes no son capaces de llegar más allá del sentido externo. Sólo el sabio alcanza el sentido interno, la verdad en sí misma. 
Resulta muy característica su exposición de las fuentes del conocimiento científico, combinando el camino intelectual, el de los sentidos y el de la tradición religiosa:

«Sabed, señores, que el hombre no debe creer en nada que no se base en uno de estos tres enunciados. Primero: Aquello que presente una clara evidencia para el intelecto humano, como ocurre con las matemáticas, la aritmética o la astronomía. Segundo: Aquello que el hombre perciba con sus cinco sentidos. Así sabe si algo es rojo o negro, etc., por el sentido de la vista, también gusta lo dulce y lo amargo, siente el frío y el calor, oye que una voz es ronca y otra aguda, puede percibir olores agradables y pestilentos, etc. Y en tercer lugar: Todas las verdades que han recibido los hombres de los profetas y de los hombres justos» . $^{3+}$

Aunque su defensa de la filosofía no es ni mucho menos ciega (puesto que muchas veces corrige sus errores y la critica), Maimónides la integra perfectamente en su edificio intelectual: en la parábola sobre el palacio real con la que concluye la Guía, Maimónides sitúa a los filósofos griegos por encima de los judíos religiosos ignorantes y de los casuistas que no se preocupan de escudriñar los principios de la religión.

«En cuanto a los que se sumergen en la especulación sobre los principios fundamentales religiosos, son los que entraban en los vestibulos donde los hombres se encuentran admitidos en grados diferentes. Quienes han comprendido la demostración de todo lo que es demostrable, han llegado a la certeza en las cuestiones metafísicas en todo aquello que sea posible o se han acercado a la certidumbre allí donde solamente es asequible la aproximación. Son los que llegaron al interior de la mansión, junto al soberano». 35

El ascenso hasta las moradas más íntimas del palacio del Rey se produce por la vía del conocimiento; estudiando la metafísica se llega al aposento mismo en el que se encuentra el Soberano, lo que no consiguen ni los juristas que sólo se dedican a la casuística, ni los fanáticos ignorantes disfrazados de religiosidad, pero sí los propios filósofos paganos; es verdad que entre los sabios que alcanzan ese grado supremo hay también categorías diversas, quedando reservado el escalón superior para los profetas. Los mayores de estos últimos, Moisés, Aharón y Miriam alcanzaron el grado máximo, el amor apasionado por Dios, llegando los tres a morir «por efecto de un beso» del mismo Dios, «en ek goce que les hizo experimentar esa comprensión y por la intensidad del amor». ${ }^{36}$

h ha-ramah, ed. S. L. Weil, p. 82.

28 Entre los numerosísimos estudios sobre el pensamiento de Maimónides véase, v.gr. D. Hartman, Maimonides. Torah and Philosophic Quest, 1976, p. 26; cf. asimismo B. Z. Bokser, «Reasen and Revelation in the Theology of Maimonides. The Conflict Between Philosophy and the Torah». HUCA 20, 1947, 541-84.

29 Guía de Perplejos, Introd.; trad. D. Gonzalo Maeso, p. 59.

30 Guía de Perplejos, Introd.; trad. D. Gonzalo Maeso, p. 59 s. 


\section{LA CÁBALA}

A la filosofía, que predomina en el pensamiento judío andalusí, se suele contraponer la mística, la Cábala, que viene de Provenza y se establece firmemente en la España cristiana, convirtiéndose para muchos en la principal reacción antifilosófica dentro del judaísmo hispano. Es frecuente relacionar el surgir de la Cábala con el ocaso del pensamiento filosófico representado por la muerte de Maimónides. Sin embargo, los mejores expertos actuales, sin negar ese hecho, destacan que las ideas que desembocan en la Cábala están presentes en el pensamiento judío, aunque de forma no articulada, desde los primeros siglos de la literatura rabíni$\mathrm{ca}^{37}$

Eso sí, el pensamiento de Maimónides, con su peculiar esoterismo, pudo provocar como reacción la cristalización de esas ideas antiguas en una línea anti-racionalista. Porque, como hemos visto, Maimónides defendía claramente la existencia de los sitre Torah, los «misterios de la Torah», y, saltándose una larga tradición centroeuropea de desarrollo esotérico de los temas del ma ăśseh běrešit o «relato de la Creación» y del ma ăśseh merkabah o «relato de la Carroza», trata de encontrar el sentido de estos viejos temas rabínicos en la filosofía aristotélica. Para los defensores de la tradición judía, esto sonaba a provocación, y no es extraño que a comienzos del s. XIII se diera al mismo tiempo el despertar de la Cábala y la polémica antimaimonidea, en la que participaron no pocos de los primeros cabalistas. El intento de Maimónides de fusionar judaísmo y filosofía significaba para ellos una novedad inaceptable, en contraste con la antigüedad y el carácter realmente judío que tenían las tradiciones de las que emergía la Cábala.

Sin embargo, y por paradójico que parezca, había coincidencias importantes entre Maimónides y los cabalistas. Se han señalado tendencias paralelas en medio de las diferencias de su sensibilidad religiosa: la búsqueda de una dimensión más profunda en los textos bíblicos, el rastreo de un orden natural que lo abarca todo, el ideal máximo del conocimiento de la ciencia divina, etc. ${ }^{38}$ No hay que olvidar tampoco que uno de los cabalistas más extremos y «extravagantes», Abraham Abulafiah (1240-1291) se sintió profundamente atraído por la Guía de Maimónides, y la comentó y enseñó con verdadero entusiasmo. ${ }^{39}$

La actitud hermenéutica de Maimónides y los cabálistas coincide en el deseo de penetrar más allá de la pura literalidad del texto, hasta los «misterios de la Torah». Pero el método de interpretación de los cabalistas, que suele llamarse sod, «secreto», es muy distinto del alegórico de los filósofos. Al no poder describir directamente la realidad de Dios y su relación con el mundo, los cabalistas recurren a un doble tipo de simbolismo que pretende representar esa realidad aludiendo a los atributos y los nombres divinos. Eso da a las palabras y a las mismas letras del texto bíblico con su puntuación una función muy especial, que permite al cabalista penetrar hasta la dimensión más profunda de la Escritura. La Torah está compuesta de nom-

31 También para Maimónides la sabiduría pertenecía en un principio al pueblo judío: «Has de saber que las numerosas ciencias que eran patrimonio de nuestra nación, para el conocimiento exacto de estas materias, se fueron perdiendo debido a la lejanía del tiempo y el dominio ejercido sobre nosotros por lós pueblos bárbaros: y tam- 
bres divinos, y es en su conjunto el nombre sublime de Dios, expresión de su plenitud de poder; es «un edificio tallado con el nombre del Santo, bendito sea», como dice 'Ezra' de Girona. ${ }^{40}$ «Se le llama Torah porque muestra y revela lo oculto y desconocido; toda la vida de lo alto está incluida en ella y nace de ella». ${ }^{41}$ "La Torah contiene todos los misterios más profundos y recónditos, todas las doctrinas sublimes manifiestas y ocultas». ${ }^{42}$ La Torah, se repite una y otra vez, es a la vez cerrada y manifiesta, oculta y patente. ${ }^{+3}$ Junto al sentido literal, tiene un significado esotérico «que sólo entienden los sabios familiarizados con los caminos de la Torah», ${ }^{4+}$ particularmente en los lugares en los que puede aludirse al «relato de la Creación» 0 al «relato de la Carroza».

\section{LA POLÉMICA ANTIMAIMONIDEA}

Ni siquiera la gran autoridad de que gozó Maimónides mientras vivió pudo librarle de las iras de los defensores a ultranza de la tradición. La disputa sobre sus puntos de vista surgiría en vida aún del filósofo; y se agudizará pocos años después de su muerte, ocurrida hacia 1232. Se debaten ante todo cuestiones concretas, como el concepto de resurrección, o más generales, como el modo de tratar el derecho judío. Pero la disensión real se da a un nivel más profundo, en el conflicto agudizado entre fe tradicional y filosofía.

El panorama de una cultura judía andalusí brillante, abierta a todo tipo de ideas, dispuesta a aceptar la filosofía, cambia sustancialmente desde mediados del s. XII. La familia de Maimónides se vería obligada a abandonar al-Andalus, y puede decirse que las comunidades judías de la España musulmana no recuperarán ya su esplendor. El esplendor de la filosofía judía, esa fórmula única de judaísmo particular de al-Andalus, insertado sin disonancias en el sistema de valores de su mundo y cultura circundantes puede darse por concluido. El clima espiritual de los reinos de la España cristiana y los problemas que preocupan a sus juderías serán muy diferentes. Los contactos teológicos, jurídicos y culturales se buscan más bien en Provenza $\theta$ entre los grandes talmudistas del Norte de Francia y el Centro de Europa, con una perspectiva mucho más orientada hacia el propio legado tradicional rabínico. Se desarrolla con fuerza la Cábala a comienzos del s. XIII, en lucha con la filosofía o llenando el vacío que ésta deja. Crecen las discordias y disputas ideológicas con los cristianos, y las presiones para lograr la conversión. El rechazo de cuanto parece ajeno al auténtico judaísmo se hace más radical. Surge el debate en el seno mismo de las juderías, a veces en función de los distintos estratos sociales, y adquiere dimensiones descomunales que alteran la vida de comunidades enteras. El anti-racionalismo se impone cada vez más, y la filosofía y las ciencias se consideran camo un grave peligro que puede desviar a cualquier judío del recto camino.

bién a consecuencia de la prohibiciôn de divulgar estas teorías a todo el mundo...», Op. cit., 1, 71; trad. D. Gonzalo Maeso, p. 196.

32 Guía. I. Intr; cf. Trad D. Gonzato Maeso, p. 63.

33 Hid: cf. Trad. D. Gonzalo Maeso, p. 64.

34 Cf. Mošeh ben Maimon, Mainónides. Sobre el Mesias. Carta a los Judios del Yenten. Sobre La Astrologia. 
Los primeros oponentes al pensamiento de Maimónides son provenzales, pero pronto se ven envueltos en ese mismo clima los principales maestros catalanes, especialmente los relacionados con el círculo cabalístico de Girona, como Yonah ben Abraham Girondí (c.12001263), Měsullam ben Šĕlomoh de Piera y Nahmánides. En la misma línea se mueven importantes maestros de Toledo como Me'ir Abulafiah, Yěhudah Alfakar o Yosef ben Todros ha-Levi Abulafiah, que aceptan las críticas provenzales contra el gran filósofo y las hacen suyas. En Toledo y otras ciudades de Castilla, la división de opiniones será manifiesta, si bien la mayor parte de los dirigentes espirituales castellanos se encuentran más cerca de las doctrinas esotéricas de la cábala y del anti-racionalismo que del pensamiento racionalista. Otros intelectuales de origen andalusí en cambio, como Šěmu'el ibn Tibbon o David Qimhi, lo mismo que los representantes de las familias aristocráticas de Aragón y Cataluña, defendían con ardor la obra de Maimónides. Los adversarios provenzales y catalanes del racionalismo tratan a toda costa de lograr que se prohíba el estudio mismo de la filosofía, en patente contradicción con lo que fuera el ideal pedagógico andalusí. ${ }^{45}$

Durante esta época cobra particular relieve la fígura de Naḥmánides (c. 1194 - 1270), prestigioso cabalista, que enseña sobre todo en Barcelona y que gracias a su ciencia talmúdica llegará a ser Rabino mayor del reino de Aragón. En la controversia sobre Maimónides buscaron su apoyo los partidarios de uno y otro bando. Si es cierto que por su carácter conservador veía peligros en la filosofía, trató de mediar en la discusión y apaciguar los ánimos. Para él, los filósofos no participaban de la sabiduría divina, dada por Dios exclusivamente a su pueblo, y por eso su búsqueda de la verdad a través de las ciencias era inútil.

«Por esto verás la crueldad del jefe de los filósofos (Aristóteles)... que niega algunas cosas que vieron muchos y que nosotros mismos vimos que eran verdad, y que se difundieron por el mundo, y que en aquellos tiempos antiguos, como en los días de Moisés, resultaban conocidas para todos, pues las ciencias en esa generación se ocupaban sobre todo de las cosas espirituales... A causa de que estaban préximos a la época de la creación y el diluvio, nadie negaba la creación ni la existencia de Dios». ${ }^{+6}$

La subordinación de las demás ciencias a la Torah es algo completamente claro para él:

«Ninguno de ellos (de los filósofos paganos) sabía acerca de la creación lo que sabe el más pequeño de Israel. Y es cosa clara que la mayor utilidad de las demás ciencias no consiste sino en servir de escala para esto, es decir, para la ciencia a la que llaman 'conocimiento del Creador' .... ${ }^{.47}$

Carta a los Judios de Montpellier. Notas biográficas, introducción, traducción y notas por Judit Targarona Borrás. Barcelona 1987, p. 237.

35. Op. cit.. II, 5I. Trad. D. Gonzalo Maese, 548.

36 Op. cit., II, 51. Trad. D. Gonzalo Maeso, 555. Véase asimismo el cap. $X$ de la sección $5^{a}$ del Libro det Conocimiento.

37. Cf. M. Idel, Mä̈monide et la mystique juive, Paris, 1991, p. 13.

38 Cf. B. Septimus, op. cit. 114. 
Los filósofos tienen que recorrer un largo camino hasta llegar a través de las diversas ciencias a conocer algo sobre la creación, y en esa cuestión, hasta su representante más destacado, Aristóteles, era incapaz de aportar pruebas sobre si el mundo es eterno o creado.

«Y mira, el más pequeño de Israel, ha leído más sobre la creación, pues ve en la Torah lo que fue creado el primer día y lo que fue creado en el segundo día, y si se fija en esto se hará experto en el tema...». ${ }^{+8}$

A pesar de que está convencido de que todo cuanto es preciso saber sobre el mundo y los fenómenos naturales, o sobre el alma, etc., se encuentra explícita o implícitamente en la Torah, y está por tanto al alcance de cualquier judío, Naḥmánides no infravalora las ciencias profanas, sino que trata de integrarlas en un lugar honorífico dentro del sistema de pensamiento judío.

Su hermenéutica parte del principio de que «la Escritura habla de cosas de aquí abajo pero alude a los temas más elevados», ${ }^{+9}$ ya que, de acuerdo con la concepción cabalística, lo que ocurre en este mundo tiene su paralelo en el mundo divino. El texto bíblico tiene dos niveles de significado, los dos verdaderos y a veces incluso coincidentes, y el cabalista puede, gracias a la tradición esotérica recibida de sus maestros, adentrarse por el «camino de la verdad» hasta la dimensión más profunda.

El ambiente antimaimonideo en Castilla, y en particular en Toledo, tiene características un tanto distintas, porque los intelectuales de este reino se consideran herederos del espíritu de alAndalus, y no rechazan de frente la razón, sino que quieren ponerla en su lugar adecuado. ${ }^{50}$ Uno de los más conocidos adversarios del racionalismo entre los dirigentes de la comunidad de Toledo, Yěhudah Alfakar, en una carta a David Qimhi, comentaba de esta manera el esfuerzo de Maimónides por conciliar la fe y la razón:

«Lo que pretendía decir es que las leyes de la naturaleza no fueron derogadas, de modo que la Torah podía compaginarse con la filosofía griega. Pensaba que una y otra eran como dos gacelas gemelas. Pero la realidad es que hay duelo y lamentos, porque la tierra no puede soportar que convivan juntas como si fueran hermanas...».5.

Como muchos de los anti-racionalistas de la época, no podía admitir tampoco la interpretación que Maimónides hacía de la Biblia, apartándose del sentido literal del texto y tratando mediante ella de imponer las categorías filosóficas sobre la letra de la Escritura.

Pero también en Toledo se dan esfuerzos de conciliación entre Maimónides y la Cábala, como el que representa Yiṣhaq ibn Lațif (ca. 1210-ca.1280). Entre los ocho grados de la sabiduría a los que debe dedicar su tiempo el hombre que desea ascender a la perfección se encuen-

39 Véase M. Idel, op. cit., 61 ss.

40 Ms. Leiden, Warner, f. 23a, citado por G. Scholem, La Cábala y su simbolismo; p. 49.

41 Zohar, III, 53b.

42 Zohar. I. 234b s.

43 Zohar: II, 230b; III 71b ss., etc. 
tran la lógica, las ciencias y la metafísica, y por encima de ellas, la ciencia de la Torah, que es «el fundamento de toda sabiduría y la raíz de toda inteligencia, y está por encima de todas»; «en ella se contienen el conocimiento y la acción por las que se apega el alma al mundo superior»; se merece que se le dedique tanto tiempo como a todas las demás juntas, ya que se trata de una «ciencia profética excelsa y única en su esencia». ${ }^{52}$

Se acerca a la Escritura como filósofo, y aunque se declara seguidor del sentido literal del texto, no duda en aceptar el alegorismo como método de interpretar los pasajes escritos en forma de parábola y de penetrar hasta los misterios de la Torah. Sin embargo, para él, la cábala supera a la filosofía pura en su naturaleza y en su efecto, ya que se ocupa de una verdad temporal, mientras que la verdad filosófica es atemporal. ${ }^{53} \mathrm{El}$ máximo conocimiento intelectual no es capaz de hacernos llegar más que a la «espalda» de Dios, pero su «cara» sólo puede alcanzarse por el éxtasis supra-intelectual, que es una experiencia superior a la de la profecía, ${ }^{5+}$ la «felicidad de la comunión suprema».

En la línea favorable a la filosofía, a pesar de tratarse de una obra menor, resulta muy representativa la 'Iggeret ha-wikkuah o «Carta de la discusión» de Šem Țob ibn Falaqera (ca. 1225-1295), discípulo de Maimónides, ${ }^{55}$ que la escribe siendo todavía joven. En la sección poética con la que comienza, dice el autor:

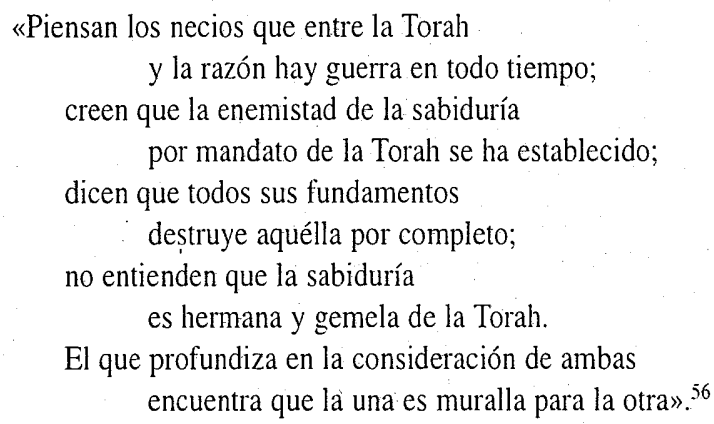

Como el resto de la obra de Ibn Falaqera, se dirige a una audiencia más amplia y sencilla que la de Maimónides, apartándose en no pocos detalles de su maestro. El autor hace una defensa clara de la filosofía y las ciencias:

44 Zohar, II, 95a.

45 En 1305, y después de muchos años de disputas. Šĕlomoh ben 'Adret y la comunidad de Barcelona lanzan el anatema contra los que estudien las obras de ciencia o metafísica de los griegos antes de los 25 años, «a fin de que esas ciencias no les seduzcan apartando sus corazones de la Torah de Israel que es superior a la sabiduría de los griegos». Véase sobre la polémica antimaimonidea D. J. Silver, op. cit; B. Septimus, op. cit., 61 ss.

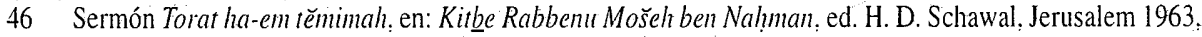
I. p. 147.

47 Op. cit., p. 155.

48 . Op. cit. p. 156.

49 Comentario al Ge 1,2. Véase E. R. Wolfson, «By Way of Truth: Aspects of Nahmanides' Kabbalistic 
«la intención de esta carta, redactada por vía de parábola y figura, es explicar en ella que el ocuparse de las ciencias verdaderas, para aquél que es digno de ellas y a quien concedió Dios en su misericordia entendimiento para descubrir sus profundidades, no está prohibido por parte de nuestra Torah, y la verdad oculta en ellas no contradice nada de nuestra fe, como piensan los simples que están vacíos de verdad y debaten sobre esto». ${ }^{57}$

El tratado tiene la forma de diálogo un tanto estereotipado entre un defensor de la tendencia tradicional, y un partidario del estudio de la filosofía. Las dos ideas fundamentales que trata de demostrar el «filósofo» al «piadoso», o judío religioso no muy erudito, son la necesidad que la religión tiene de la filosofía y el acuerdo estrecho que se da entre la Torah y la filosofía o ciencia. ${ }^{58} \mathrm{El}$ piadoso abre el diálogo acusando al filósofo de mezclar «con las palabras rectas de la Torah las palabras de los herejes y sectarios», expresando así el rechazo general de la filosofía por parte de los judíos tradicionales.

El estudioso demuestra que los filósofos no son necesariamente herejes, pero no sostiene que la filosofía sea infalible, sino que adopta un postura moderada: se toma de la filosofía lo que es verdadero y está de acuerdo con la religión; hay que comer la granada y arrojar la piel. Hay que aprender la verdad de todos, incluso de los que niegan a Dios. La Torah recoge como algo positivo el consejo que diera Jetro, no judío, a Moisés (Ex 18).

«el hombre tiene que aprender la verdad de los que la dicen, aunque no sean creyentes, lo mismo que se coge la miel de las abejas; sobre todo teniendo en cuenta que esos filósofos no niegan al Creador ¡bendito sea! ni su gloria, sino que creen en Él y en su unicidad, y reconocen que 'Él es Dios arriba en los cielos y abajo sobre la tierra' (De 4,39) y que 'no hay otro fuera de Él' (De 4,35)». ${ }^{59}$

En la línea del Kalam árabe, Šem Tọ sostiene que el que cree en Dios sólo por la tradición, es inferior a quien cree por demostración racional.

«Si un hombre experimentado, que sabes que no te engañará bajo ningún concepto, te dice acerca de alguna cosa que existe y que él la ha visto, y tú nunca la has visto, y testifican también sobre eso después de él hombres experimentados y fidedignos, a pesar de que tu fe en lo que dicen es fe firme, sería más sólida si la hubieras visto con tus propios ojos; e igualmente, será más firme tu fe si la alcanzas por tu entendimiento que si la obtienes por la tradición». ${ }^{60}$

Hermeneutic», AJSReviel1; 14, 1989: 103-78, particularmente p. 110.

50 Cf. B. Septimus, Op. cit., 94 ss.

51 A. Lichtenberg, ed., Qobeș tě "nobotha-RaMbaM wě-'iggerotaw; Leipzig. 1859, III, 2a.

52 Comentario a Qo: pp. $52 \mathrm{~s}$.

53 Cf. Rạb pe alim, n. 39.

54 Cf. Ginze ha-melek: cap. 37; 41.

55 La obra fue impresa por Yișhạ `Aqriš. Constantinopla 1577, por Gersom ben Beșalel Kohen, Praga 1610 
Al hombre que desea llegar a la verdad y la perfección no le basta el argumento de la tradición, sino que tiene que penetrar con su entendimiento en los fundamentos y principios de la realidad. El filósofo demuestra al piadoso que en la Torah, los Profetas y los Escritos se alude a «temas profundos» relacionados con las ciencias y la filosofía, y que sólo pueden entenderse mediante estas últimas. ${ }^{61}$

«Lejos de mí comparar a unos con otros, pues sé que lo alcanzado por los profetas ique descansen en paz! está muy por encima de lo captado por cualquier otro, y los filósofos mismos lo mencionaron; y también sé que lo que alcanzaron los filósotos de la metafísica y la ciencia de la naturaleza es muy poco, y en ese poco hay bastantes dudas. Ellos reconocen todo esto. Y yo no tengo otra intención que la de explicarte que la verdad mencionada en esas ciencias no es lo contrario de nuestra Torah para quienes descubren lo profundo de la intención de la Torah y conocen esas ciencias en su verdad, como piensas tú y cuantos no aprendieron otra cosa que la Torah». ${ }^{62}$

Con una postura abierta, similar a la de Maimónides, el filósofo se declara universalista en la búsqueda de la verdad:

«y no hay diferencia si esos antiguos eran de nuestra nación o no; pues si el estudio se hace empleando un método verdadero y libre de toda falta, no nos preocupa si son de nuestra nación o no. Y puesto que esos filósofos antiguos se pasaron la vida investigando las pruebas verdaderas y distinguiéndolas de las no verdaderas, y se esforzaron en separar unas de otras, es preciso que si queremos alcanzar las cuestiones mediante el entendimiento estudiemos los libros que ellos escribieron sobre este tema, y que lo que haya en ellos de verdad lo aceptemos, y lo que no sea verdad y esté en contradicción con las palabras de nuestra Torah, lo arrojemos lejos, y no discurramos sobre ello», ${ }^{63}$

\section{LA FILOSOFÍA Y LAS CIENCIAS}

«son las que llevan a la perfección la forma específica suya, que es el entendimiento, y gracias a èas ciencias verdaderas pasa su entendimiento de la potencia al acto, y domina la verdad de las cosas creadas, y al dominar su verdad alcanza a su Creador, y en el grado en que lo alcanza se acerca a Él y se convierte en piadoso del Señor». ${ }^{6+}$

y por A. Jellinek, Viena 1875. Las tres ediciones son defectuosas, como hicieron notar, entre otros, Y. Dan, Oș.Yĕh.Sĕf. 6, 1963, 42-6, y G. Dahan, «Epistola Dialogi. Une Traduction latine de l'Igeret ha-1ikual! de Shemtov ibn Falaqera. Étude et Édition». Sefarad, 39, 1979, 48 s., n.8. S. Harvey ha preparado una edición crítica con trad. inglesa, Falaquera's Epistle of the Debate. An Introduction to Jewish Philosophy; Harvard. 1987. Nos atenemos al 
La misma idea aparece en otras obras de Ibn Falaqera: la fe y la razón son interdependientes: la razón puede verificar las verdades de la religión, y una fe razonada es más fuerte que la que se basa sólo en la tradición. La fe perfecciona la razón:

«El intelecto brilla gracias a la luz de la fe, y no alcanza su perfección si no es gracias a ella». 65

Pero, coincidiendo también en este tema con Maimónides y con muchos pensadores musulmanes, la filosofía no es para las masas ni las gentes incultas, que podrían recibir daño de ella:

«Has de saber, oh piadoso, que esas ciencias, y en particular la metafísica, no puede soportarlas la mente de todos los hombres, e igual que el pan y la carne no los soporta el cuerpo de los pequeños ni de los enfermos, sino sólo el de los sanos, tampoco puede soportar esas ciencias a no ser una mente muy apropiada y un alma habituada desde el principio a las buenas virtudes y ejercitada en las ciencias especulativas que tienen como finalidad ejercitar el alma; e incluso las mentes perfectas no tomarán de ellas sino un témino medio según su capacidad. Por eso se comparan con la miel, que hasta el cuerpo sano si come de ella más de la medida la vomita, y puede ser la causa de que su temperamento se incline hacia uno de los extremos». ${ }^{66}$

Los filósofos no judíos eran en realidad «gentes de bien» que buscaban la verdad y la perfección, muy próximos en no pocas cosas a los principios del judaísmo.

«Sabe que para ellos el fin último consiste en asemejarse a Dios ¡bendito sea! según la capacidad humana». ${ }^{67}$

Ante esas palabras, el judío tradicionalista reconoce:

«Me parece que eso está próximo a lo que se dice en nuestra Torah sobre el amor al Señor tu Dios y el apegarse a Él». ${ }^{68}$

En todo caso, y en la línea de su maestro, Maimónides, la Escritura contiene doctrinas esotéricas que sólo pueden entenderse mediante el conocimiento de la ciencia:

«Sabe que sólo conociendo las verdaderas ciencias puede el hombre entender los secretos y misterios de la Torah». ${ }^{69}$

texto hebreo de esta última edición.

56 Ed. Harvey. p. 55; la expresión de que la sabiduría es hermana de la religión se encuentra de modo similar en Averroes: como observa el propio Harvey; op. cit. p. 14.

57 Op. cit. p. 56.

58. Se ha señalado con razón que el tratadito tiene no pocos puntos tomados del Fașl al-maqãl de Averroes. 


\section{LOS SIGLOS XIV-XV}

Los últimos siglos de presencia judía en la Península no hacen sino acentuar la problemática de confrontación con el cristianismo y, como consecuencia, de mayor afirmación de la propia tradición y rechazo de los elementos ajenos, o, lo que es lo mismo, suponen un reforzamiento de la línea anti-racionalista. Eso no significa, desde luego, que Maimónides y la filosofía no tuvieran también sus defensores en estos tiempos críticos; Yosef ben Šem Tob ibn Šem Tọb, su hermano Yiṣhaqu, su hijo Šem Tọb, Abraham Bibago, etc. son representantes más o menos decididos del aristotelismo, aunque oscurecidos por la mayor categoría y difusión de las ideas de sus adversarios. Mencionaremos solamente algunas de las figuras más destacadas en la historia del pensamiento.

Yosef ibn Waqar, filósofo y cabalista de Toledo de la primera mitad del s. XIV, escribe un significativo Tratado de la conciliación entre la filosofía y la religión en el que busca una solución de compromiso entre los métodos empleados por los que se dedican a la Cábala, aferrados a la tradición, y los filósofos, basados en la argumentación; el toledano se inclina del lado de la primera. Como muestra de su sentido de la moderación, trata sin embargo de que la Cábala quede penetrada por la razón, sin que su solución resulte aceptable para los unos ni para los otros. ${ }^{70}$

Hasday Crescas (1340-1412) es un notable representante de la línea anti-racionalista que se desarrolla en Cataluña, en medio de las dificultades que asedian al judaísmo peninsular en los últimos años del s. XIV y comienzos del XV. Ve como adversarios a la vez a los aristotélicos y a los cristianos. Para él, Maimónides se equivocó en su planteamiento fundamental: a Dios no se llega por la vía del conocimiento, sino por la del temor y el amor. Además, trató de sustituir la ciencia tradicional del pueblo judío, perdida a causa de sus tribulaciones en el exilio, por la ciencia de otros pueblos, así que su trabajo comenzará por demostrar los errores de la física de Maimónides y Aristóteles. Su primer interés consiste en demostrar que la imagen aristotélica del universo expuesta por Maimónides era falsa, y por tanto falso también el tipo de judaísmo que sobre ello trató de levantar el pensador cordobés. ${ }^{71}$

La figura de Yěhošua ha-Lorqi (ca. 1350-ca. 1419) es una muestra patente de las tensiones y contradicciones en que debieron moverse los judíos hispanos durante los años críticos que van de los disturbios de 1391 a la Disputa de Tortosa de 1413-14. La conversión de su amigo Šĕlomoh ha-Levi, Pablo de Santa María, le haría reflexionar profundamente sobre el tema de la fe y la razón. Esta última se convierte para él en el criterio único para que el hombre decida cuál es la verdadera religión. El hombre religioso está obligado a tratar de conocer las bases de su religión y decidir si es o no la verdadera. De esta forma, la filosofía le lleva

pero hay una diferencia bastante significativa: para el filósofo árabe, la Ley religiosa recomienda o hace obligatorio el recurso a la filosofía, mientras que para el judío, la filosofía es necesaria para llegar a practicar como es debido la religión. 
hacia el cristianismo: adopta el nombre de Jerónimo de Santa Fe e interviene activamente en las disputas públicas con las que se trata de convertir a los judíos. ${ }^{72}$

La atmósfera de los años que restan del s. XV es decididamente contraria a la razón y la filosofía. Yișhaqa Arama (1420-1494), en la misma línea de Crescas, y en último término de Yěhudah ha-Levi, critica a Maimónides y el aristotelismo, y defiende que la verdad religiosa está por encima de la razón humana, llena de limitaciones. La filosofía no permite llegar al fin último del hombre ni a la felicidad suprema. La religión no puede basarse en demostraciones, ni la fe puede asentarse sobre el entendimiento. Hay contradicciones entre la fe y la razón que no pueden disimularse como quería Maimónides, y no se debe interpretar la Escritura alegóricamente, salvo en casos contados. Cuando los argumentos racionales están en contra de la tradición religiosa, es esta última la que tiene razón. Los judíos son el pueblo de la revelación, y no necesitan la especulación filosófica para llegar a la verdad. ${ }^{73}$

Otro caso notable de anti-racionalismo es el de uno de los últimos intelectuales de prestigio del judaísmo hispano antes de la expulsión y en el momento en que ésta tiene lugar: Yișhaq 'Abravanel (1437-1508). Comenta la obra de Maimónides, y con frecuencia se opone a sus ideas, con una mezcla de respeto y crítica. Para él es evidente la primacía de la fe sobre la razón; la revelación es la guía de nuestro pensamiento y el objetivo de nuestra razón, la única vía posible del conocimiento humano. En su acercamiento a la Biblia, y en sus numerosos Comentarios busca el sentido literal del texto, sin renunciar a ver «lo que esta secretamente implícito en él»; de esa forma trata de construir todo un sistema coherente basado exclusivamente en la revelación. ${ }^{74}$ Con él se cierra de algún modo el ciclo del pensamiento judío medieval, inclinándose significativamente hacia la crítica de la razón y la filosofía, más propia del Medievo que del Renacimiento que florece en la Europa en la que le ha tocado vivir.

\section{BIBLIOGRAFÍA SELECTA SOBRE EL TEMA}

A. J. Arberry, Revelation and Reason in Islam, Londres, 1957.

I. E. Barzilay, Between Reason and Faith, La Haya-París, 1967.

B. Z. Bokser, «Reason and Revelation in the Theology of Maimonides. The Conflict Between Philosophy and the Torah», HUCA 20, 1947, 541-84.

T. A. M. Fontaine, In Defence of Judaism: Abraham Ibn Daud. Sources and Structure of ha-Emunah haRamah, Assen/Maastricht, 1990.

E. Gilson, Reason and Revelation in the Middle Ages, New York, 1938.

D. Hartman, Maimonides. Torah and Philosophic Quest, 1976.

M. R. Hayoun, L'exégèse philosophique dans le judaïsme médiéval, Tübingen 1992, 1-24.

M. Idel, Maïmonide et la mystique juive, Paris, 1991.

59 Op. cit., p. 58.

60 Op. cit., p. 60.

61 Op. cit., pp. 69 ss.

62. Op. cit. p. 70. 
J. Lomba, La Filosofía islámica en Zaragoza, 1987.

H. de Lubac, Exégèse Médiévale. Les quatre sens de l'Écriture, Lyon, 1959.

Mošeh ben Maimon, Maimónides, Guía de Perplejos, Introd., trad. D. Gonzalo Maeso, Madrid, 1984. Mošeh ben Maimon, Maimónides. Sobre el Mesías. Carta a los Judíos del Yemen. Sobre la Astrología.

Carta a los Judíos de Montpellier: Notas biográficas, introducción, traducción y notas por Judit Targarona Borrás, Barcelona 1987.

B. Netanyahu, Don Isaac Abravanel, Statesman and Philosopher, Filadelfia 1953, 95 ss.

Ch. Pearl, The Medieval Jewish Mind, 1971.

A. Sáenz-Badillos, J. Targarona, Diccionario de Autores Judios (Sefarad. Siglos X-XV), El Almendro, Córdoba, 1988.

A. Sáenz-Badillos, J. Targarona, «La 'ciencia de los griegos' en los judíos de la España medieval», Homencije al Dr: L. Gil Fernández, (en prensa).

A. Sáenz-Badillos, «La exégesis de la creación y el paraíso en la obra de Selomoh ibn Gabirol», en:

Palabra y vida. Homenaje a Jose Alonso Diaz en su 70 cumpleaños, ed. A.Vargas-Machuca y G.Ruiz, Madrid, 1984, 369-382.

J. Sarachek, Faith and Reason. The Conflict over the Rationalism of Maimonides, Williamsport, 1935.

J. Schlanger, La Philosophie de Salomon Ibn Gabirol, Leiden, 1968.

B. Septimus, Hispano-Jewish Culture in Transition. The Career and Controversies of Ramah, Cambridge/Mass.

D. J. Silver, Maimonidean Criticism and the Maimonidean Controversy, 1180-1240, Leiden, 1965.

C. Sirat, La Philosophie Juive au Moven Age, Paris, 1983.

C. Sirat, «Les philosophes juifs d'Espagne au Moyen Age et leur rapports avec la philosophie arabe et chrétienne», REJ 144, 1985, 51 ss.

B. Smalley, The Study of the Bible in the Middle Ages, Oxford, 1952.

L. D. Stitskin, Judaism as a Philosophy: The Philosophy of Abraham Bar Hiyya (1065-1143), New York 1960.

F. Talmage, «David Kimhi and the Rationalist Tradition», HUCA 39, 1968, 177-218.

Ch. Touati, «La controverse de 1303-1306 autour des études philosophiques et scientifiques», REJ, 127, 1968, 21-37.

J. Targarona, «Pensadores judíos cordobeses de los ss. XI-XII: Yosef ibn addiq, Abraham ibn Daud y Mošeh ben Maimon», en: Los Judíos y Lucena, ed. J. Peláez, Córdoba, 1988, 49-64.

G. Vajda, La teología ascética de Balỵa ibn Paquda, Madrid-Barcelona, 1950.

G. Vajda, Recherches sur la Philosophie et la Kabbale dans la pensée juive du Moren Age, 1962.

E. R. Wolfson, «By Way of Truth: Aspects of Nahmanides' Kabbalistic Hermeneutic», AJS Review, 14, 1989, 103-78.

H. A. Wolfson, Crescas' Critique of Aristotle. Problems of Aristotle's Physics, 1929.

Angel Sáenz Badillos

Dpto. de Hebreo

Facultad de Filología

Universidad Complutense

E-28040 MADRID 